\title{
Large Scale Cardiovascular Model Personalisation for Mechanistic Analysis of Heart \& Brain Interactions
}

\author{
Jaume Banus ${ }^{1}$, Marco Lorenzi ${ }^{1}$, Oscar Camara $^{2}$, and Maxime Sermesant ${ }^{1}$ \\ 1 Inria, Epione team, Université Côte d'Azur, Sophia Antipolis, France \\ jaume.banus-cobo@inria.fr \\ 2 PhySense, Department of Information and Communication Technologies, \\ Universitat Pompeu Fabra, Barcelona, Spain
}

\begin{abstract}
Cerebrovascular diseases have been associated with a variety of heart diseases like heart failure or atrial fibrillation, however the mechanistic relationship between these pathologies is largely unknown. Until now, the study of the underlying heart-brain link has been challenging due to the lack of databases containing data from both organs. Current large data collection initiatives such as the UK Biobank provide us with joint cardiac and brain imaging information for thousands of individuals, and represent a unique opportunity to gain insights about the heart and brain pathophysiology from a systems medicine point of view. Research has focused on standard statistical studies finding correlations in a phenomenological way. We propose a mechanistic analysis of the heart and brain interactions through the personalisation of the parameters of a lumped cardiovascular model under constraints provided by brain-volumetric parameters extracted from imaging, i.e: ventricles or white matter hyperintensities volumes, and clinical information such as age or body surface area. We applied this framework in a cohort of more than 3000 subjects and in a pathological subgroup of 53 subjects diagnosed with atrial fibrillation. Our results show that the use of brain feature constraints helps in improving the parameter estimation in order to identify significant differences associated to specific clinical conditions.
\end{abstract}

Keywords: 0D model · Cardiovascular modelling · Personalisation · White matter damage $\cdot$ Brain damage $\cdot$ Atrial fibrillation

\section{Introduction}

Cerebrovascular diseases are related to a variety of heart diseases such as heart failure [1] or atrial fibrillation (AF) [2], sharing several risk factors such as cholesterol, diabetes or high blood pressure. In parallel, it has been shown that stroke doubles the risk of dementia [3]. All these connections suggest a common underlying pathological process that links cardiac function with brain atrophy. Large scale analysis on databases combining cardiovascular and brain data from 
the same individuals are thus required to demonstrate and better understand the interaction between brain and heart. To this end, studies such as the UK Biobank aim at the acquisition of multi-modal databases containing both heart and brain imaging information [4]. Thanks to these databases ongoing studies have focused on the study of the relationships between cardiovascular risk factors and image-derived features, such as subcortical volumes [5]. However, a number of fundamental descriptors of the cardiac function are not possible to obtain in-vivo, i.e: heart contractility or fibers stiffness. Personalised modelling approaches allow us to estimate these descriptors and gain insight of the cardiac function, allowing us to obtain more reliable results and relate them to brain damage information.

Personalizing a cardiovascular model for a given subject is an ill-posed problem that implies estimating the model parameters so that the simulation behaves as close as possible to the available clinical data. In this work we will focus on a 0D model of the whole cardiovascular system. Previous studies have used multiscale models to describe the whole-body circulation and study the venous blood flow in the brain [6]. However, their modeling of the heart chambers does not take into account the contractile and elastic properties of the heart. While other models of the whole-body circulation are available $[7,8]$, to our knowledge, no explicit modelling study relating neurodegeneration and cardiovascular parameters has been done.

In this paper we aim to study the relationship between cardiovascular indicators and brain volumetric features extracted from the imaging data available in UK Biobank, through the personalisation of a cardiovascular lumped model using the approach presented in [9]. The use of this approach allows us to tackle the ill-posedness nature of the personalisation and identify plausible and coherent solutions across the population. To achieve that, we define a regularisation term that can be extended to take into account features not present in the lumped model, allowing to explore the effect of including brain features as additional constraint. We apply this framework to a large cohort composed by more than 3 000 subjects for which cardiac and brain information was jointly available in the UK Biobank. To illustrate how to exploit the framework to identify meaningful clinical relationships, we applied it in a subset of subjects diagnosed with AF, which is considered as an independent risk factor for stroke and dementia $[2,10]$. We identified statistically significant associations between the personalised model parameters and brain volumetric features that match findings reported in previous clinical studies.

The paper is structured as follows: in section 2.1 we detail the data preprocessing and inclusion criterion for the whole-population analysis. Following, in section 2.2 we present the lumped model and how to take into account the subject's information to constrain the solution space in the personalisation. Next, in section 2.3 we assess the impact of our model in determining significant relationships between the estimated cardiac parameters and brain damage using the AF subset. Finally, in section 3 we present the obtained results. 


\section{Methods}

\subsection{Data pre-processing and inclusion criterion}

Our analysis includes data from UK Biobank participants for which all brain image modalities and all cardiac-image derived indicators were available, for a total of 3783 subjects. In the available cardiac images it was possible to quantify the cardiac function using indicators such as stroke volume (SV), cardiac output $(\mathrm{CO})$ or ejection fraction (EF). Multi-modal brain MRI images allowed the extraction of image-derived features such as brain tissue volumes and white matter hyperintensities (WMHs), one of the most common indicators used to assess neurological damage.

Using FLAIR MR images, WMHs were segmented by the lesion prediction algorithm (LPA), available in the lesion segmentation toolbox (LST) [11] for $\mathrm{SPM}^{3}$. FLAIR MR images were pre-processed following the protocol described in [12], in which gradient distortion correction and defacing were performed. After discard subjects for which pre-processing (449) or segmentation of WMHs (250) failed, the final number of available subjects was 3084 . From the segmentations we extracted the total volume of WMHs and the number of lesions. All brainrelated volumes were normalized by head size.

\subsection{Cardiovascular lumped model}

The cardiovascular personalisation of the subjects was performed by using the 0D model shown in Figure 1 which is a simplification of a 3D cardiac electromechanical model [13] derived in [14]. In the 0D version, which assumes spherical symmetry, the myocardial forces and motion can be described by the inner radius $\left(R_{0}\right)$ of the ventricle. Deformation and stress tensors are also reduced to $0 \mathrm{D}$ forms, which allow us to characterise the heart contractile $\left(\sigma_{0}\right)$ and elastic $\left(C_{1}\right)$ properties of the heart.

The model $M$ consists in a set of ordinary differential equations with $P_{M}$ parameters, e.g. maximum contraction of the heart fibers or its stiffness. The state variables of the model are denoted by $O_{M}$, e.g. arterial or venous pressures, and they describe the state of the system. During the personalisation we are interested in a subset of $n$ state variables, such that $O=\left(O_{1}, O_{2}, \ldots, O_{n}\right)$, and we vary a subset $\theta$ of the $P_{M}$ model parameters. We consider $O(\theta)$ the set of state variables generated by the model for a given set of $\theta$. The goal is to find $\theta *$ such that $O(\theta *)$ best approximates the target features $\hat{O}$.

Due to the high dimensionality and non-convexity of this inverse problem, we solve it with the CMA-ES optimization algorithm based on evolution strategies [15]. CMA-ES minimizes a given error function by combining maximum likelihood principles with natural gradient descent on the ranks of the point scores (i.e: the score of each individual at every generation). The error function $S(\theta, \hat{O})$ is defined as the $L_{2}$ distance between $O(\theta)$ and $\hat{O}$. Since each target feature has different range of values we defined a tolerance interval, Tol, for each

\footnotetext{
${ }^{3}$ https://www.fil.ion.ucl.ac.uk/spm
} 


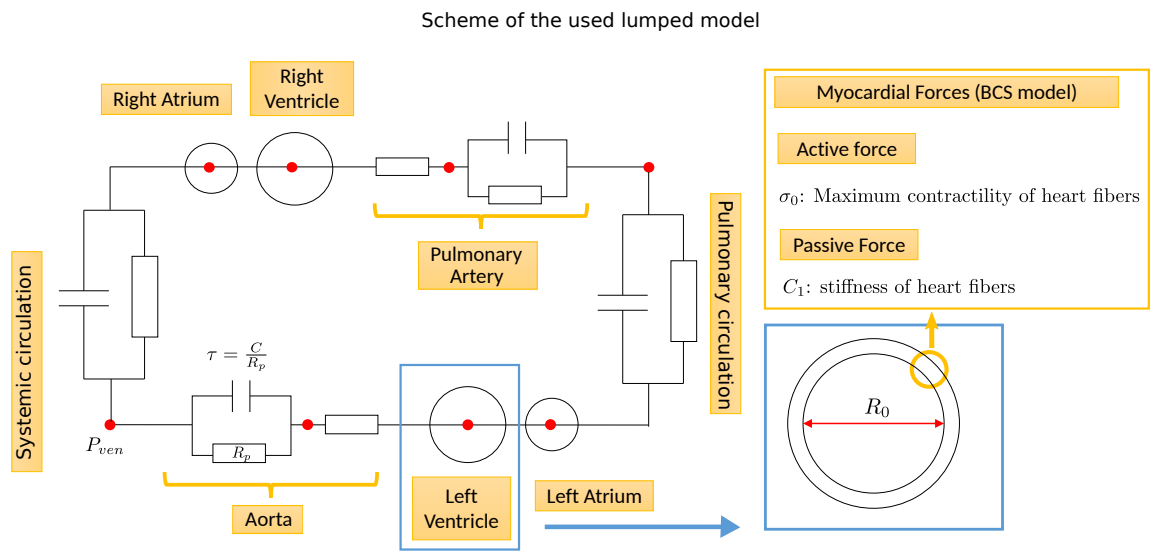

Fig. 1. Simplified schematic representation of the lumped model showing the parameters used in the personalisation. The $0 \mathrm{D}$ representation of the myocardial forces has been omitted for the sake of clarification. $\tau$ characterizes the contractility of the aorta, $R_{p}$ the peripheral resistance and $P_{v e n}$ the venous pressure.

feature $i$ to be able to compare the different outputs. This can be formalized as shown in Equation 1:

$$
S(\theta, \hat{O})=\sum_{i=1}^{n} \frac{\left(O_{i}(\theta)-\hat{O}_{i}\right)^{2}}{T o l_{i}}
$$

Based on the available clinical data, we selected the following target features for the personalisation; stroke volume (SV), ejection fraction (EF), diastolic blood pressure (DBP), mean blood pressure (MBP) and end-diastolic volume (EDV). Considering the uncertainty of the measured data, the tolerance interval for each feature was set to $10 \mathrm{ml}$ for the SV and the EDV, $200 \mathrm{~Pa}$ for the DBP and the MP, and $5 \%$ for the EF. Finally, the personalized parameters of the cardiovascular model were maximum contraction of the heart fibers $\sigma_{0}$, stiffness of the heart fibers $C_{1}$, peripheral resistance $R_{p}$, venous pressure $P_{v e n}$, and the characteristic time $\tau$ of the aorta, which defines the time that takes for blood pressure to decrease from systolic to the systemic, or "asymptotic" value. We selected these parameters based on a sensitivity analysis in which we assessed the influence of each parameter over the selected target features.

Since the solution of equation 1 is non-unique, there is an observability difficulty in this personalisation problem. To tackle this issue, we used the iterativeupdate prior (IUP) approach presented in [9] to introduce constraints in the fitting process. In the IUP method a regularization term, $R(\theta, \mu, \Sigma)$, is used to reduce the variability in the estimation of the parameters. The regularization constrains the directions in which we explore the parameter-space by using the relationships among the model parameters. Formally, the regularization term is 
parameterized by an expected value $\mu$ and by a covariance matrix $\Sigma$ encoding the relationships across parameters.

$$
R(\theta, \mu, \Sigma)=(\theta-\mu)^{T} \Sigma^{-1}(\theta-\mu) .
$$

Therefore, the fitting score becomes:

$$
S(\theta, \hat{O}, \mu, \Sigma)=S(\theta, \hat{O})+\gamma R(\theta, \mu, \Sigma),
$$

where $\gamma$ defines the relative importance of the regularization term. This term is updated at each IUP iteration, using the obtained mean value of the fitted parameters and the estimated covariance in the previous iteration.

Accounting for brain information in the $0 \mathrm{D}$ model. $R(\theta, \mu, \Sigma)$ can be extended to incorporate relationships with features not present in the cardiovascular model. In our setting, we included in the regularization term the extended feature space corresponding to the concatenation of the model parameters, $\theta$, with the brain and clinical information, here denoted by $\phi$. We used the total brain volume, the ventricles volume, the obtained WMHs features, age, sex and body surface area (BSA). Therefore, the problem in equation 3 becomes:

$$
S(\theta, \hat{O}, \mu, \Sigma, \phi)=S(\theta, \hat{O})+\gamma R(\theta, \phi, \mu, \Sigma) .
$$

Equation 4 now accounts for a covariance term constraining the parameters according to the extended set of information. We have used 10 IUP iterations and assessed the results at different $\gamma$ levels $(0.1,0.5,2$ and 10). The optimisation is performed over the logarithm of the parameter values.

\subsection{Atrial fibrillation analysis.}

Considering the dataset obtained after the pre-processing described in section 2.1 we had access to 53 subjects diagnosed with AF. Using bootstrapping we sampled 100 control groups of the same sample size of the AF group and without any significant difference in age, sex and BSA. The sampled controls came from the subset of subjects without any diagnosed cardiovascular disease $(n=2022)$. We applied the framework described in the previous section to each bootstrap subset composed by the AF group and sampled control group, to obtain the bootstrap distributions of the correlations between cardiac and external parameters. This approach allowed us to exploit the dataset variability for assessing the difference between cardiac and brain associations.

\section{Results}

Whole-population analysis. As expected, we observed that as the value of $\gamma$ is increased (i.e. more regularisation), the fitting error increases and at the same time the number of outliers is reduced and the estimated distributions 
a)
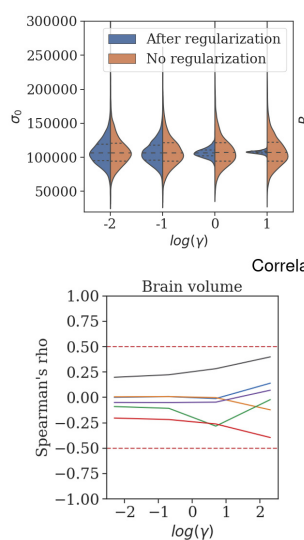

Correlations of mode

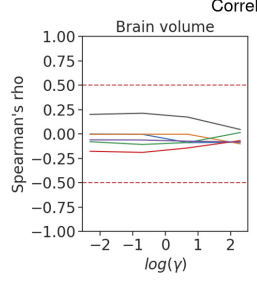

d)
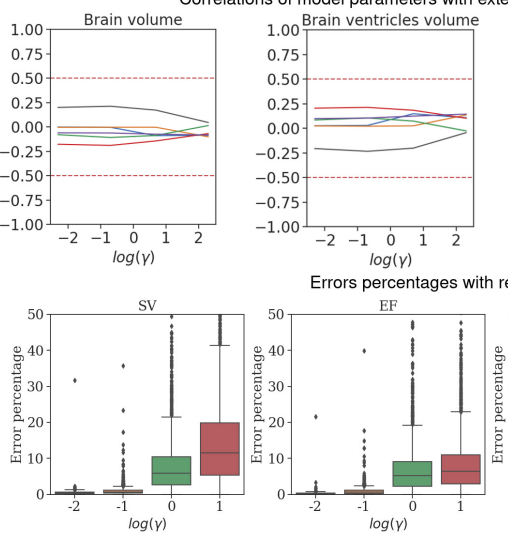

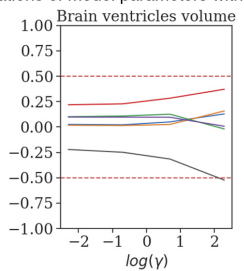

Estimated parameters distributions: effect of regularization

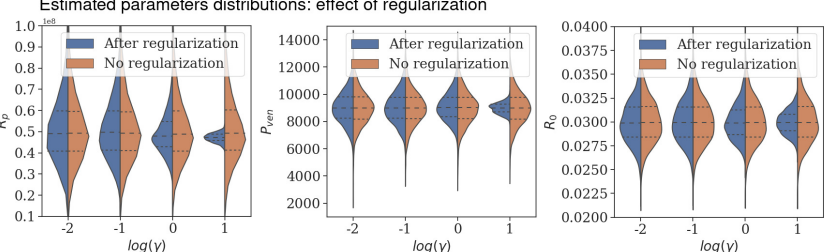

${ }^{-1}{ }_{\log (\gamma)}{ }^{0}$
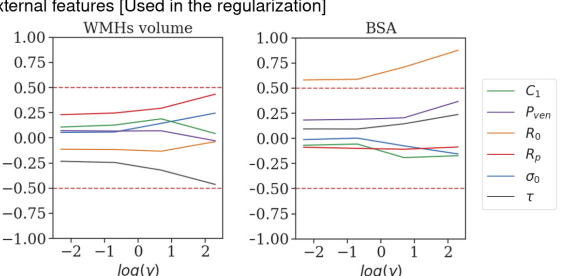

$\log (\gamma)$

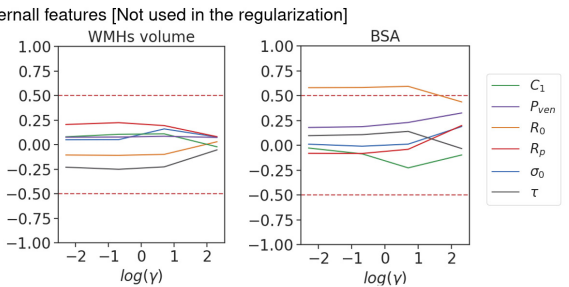

Errors percentages with respect to the target features
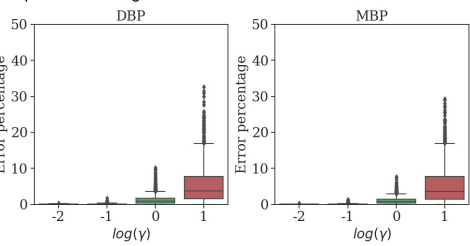

Fig. 2. a) Estimated density distributions of the fitted parameters at different regularization levels. Initial and final distributions after 10 iterations in brown and blue respectively. The variability among the initial distributions is due to the variability in the sampling that CMA-ES performs during the optimization.Error b) Evolution of Spearman's rank correlation coefficient between the model parameters and the external parameters as the regularization level increases when external features are considered in the regularization and c) when external features are not considered. Model parameters being: maximum heart fibers contractility $\sigma_{0}$, heart fibers stiffness, $C_{1}$, left ventricle size $R_{0}$, peripheral resistance $R_{p}$, aorta characteristic time $\tau$, and venous pressure $P_{v e n}$. d) Error percentages with respect to the target features. Stroke volume (SV), ejection fraction (EF), diastolic blood pressure (DBP) and mean blood pressure (MBP). While end-diastolic volume (EDV) is not shown due to space issues, its error pattern was similar to the one observed in the SV

have lower variability, as can be seen in Figure $2 \mathrm{a}$. We can observe that strong regularisation even shrinks some parameters close to a constant value, implying that those parameters cannot be observed from the available data. Looking at the correlation of the model parameters with the external features we note the 
strong correlation between the left ventricle size, $R_{0}$, and the $\mathrm{BSA}$, even for low $\gamma$ values. Moreover, there is a positive correlation between peripheral resistance, the WMHs volume, and brain ventricles volume, which are at the same time negatively correlated to the aorta characteristic time, $\tau$. The number of WMHs lesions and age followed the same correlations pattern, but due to space issues they have been omitted. On the other hand, brain volume is positively correlated with $\tau$ and peripheral resistance. An increase in peripheral resistance can be associated to higher DBP, while a decrease in contractility, $\tau$, can be interpreted as an increase in arterial stiffness leading to high SBP. Both, DBP and SBP, have been previously associated to WMHs [16]. In figure $2 \mathrm{~b}$ we note that the significant correlations present when no regularization is applied become stronger as regularization increases, while the non-significant correlations stay close to zero. This behavior is expected since regularization is constraining the space of feasible solutions. Therefore, as we increase $\gamma$ we further limit the feasible parameter-space towards the set of solutions that satisfy the existing relationships between the parameters. In figure $2 \mathrm{c}$ we observe the obtained correlations when the external features are not taken into account. In this case, the solutions are constrained into a different parameter-space in which the relationships between the model parameters and the external features are lost. Limiting the interpretability when assessing the parameters estimations with respect external factors not present in the mechanistic model.

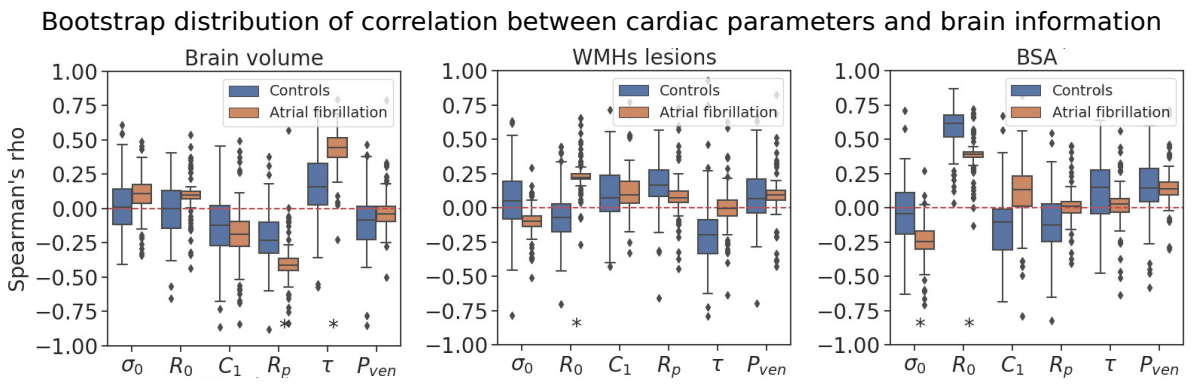

Fig. 3. Comparison of the Spearman's rank correlation coefficient bootstrap distributions obtained at $\gamma=0.5$ between the personalised model parameters and the external features. Blue boxplots correspond to control groups and brown to AF subjects. * denotes that the correlations are significantly different according to the Wilcoxon ranksum test, and that in the AF group correlations are significantly greater or smaller than 0 ( $5 \%$ significance level).

Atrial fibrillation. In Figure 3 we observe a statistical description of the empirical distribution of correlations obtained from the bootstrap analysis done in the AF subset. The results are obtained with trade-off $\gamma=0.5$, which in the whole-population analysis provided a good a balance between data-fit and 
regularization. We assessed the difference between the controls and AF groups correlations distributions using the Wilcoxon rank-sum test with a significance level of $\alpha=0.05$. Moreover, to consider the obtained results as significant we assessed if in the AF group the obtained correlations were statistically greater or smaller than 0 with a $5 \%$ significance level.

In the brain volume we observe the same correlations found in the wholepopulation analysis, but it can be seen that for the AF group these correlations are stronger, which suggests that in the AF subjects brain is more susceptible to cardiovascular factors. For the BSA we found the positive correlation with the left ventricle size observed in section 3 and a negative correlation with the maximum heart contractility $\sigma_{0}$. In the number of WMHs lesions we can observe a positive correlation with the left ventricle size. The associations of BSA with $\sigma_{0}$ and WMHs with $R_{0}$ could be related to cardiac dilation due to an increased impairment of the functioning heart in AF. Interestingly, in the AF control subjects the correlation between WMHs and left ventricle size is negative. These findings suggest an association between AF and WMHs. Moreover, they agree with previous studies reporting an association between left ventricle remodelling and AF [17]. No significant associations were found for brain ventricles volume, WMHs volume and age.

\section{Conclusions}

We have modeled 3084 subjects with a 0D cardiovascular model and we constrained the available parameter-space during personalisation by incorporating external features in the regularization term, allowing us to study their influence in the estimated model parameters. The use of this approach gives access to a generative model that allows to analyze the relationships between external features and non-observable parameters such as the characteristic time of aorta, $\tau$, which we found to be related with brain-volumetric features. Using the same framework we assessed a clinical subgroup in which we have found meaningful clinical relationships, linking AF with WMHs and heart remodelling. Our model does not currently simulate the cerebral blood flow, while previous studies [6] suggest that WMHs are due to more localize vascular impairments. This highlights the need to obtain a local flow characterization to estimate more relevant parameters. Moreover, the presented approach can be seen as a parameter selection approach. It allows to identify which parameters cannot be estimated from the available data and find a parameter subspace of solutions in which the non-observable parameters get close to constant values. The identification of the non-observable parameters coupled with human modelling expertise can help in the selection of a reduced subset of observable cardiovascular parameters for personalisation. Future work will go towards the local blood flow characterization in the brain, as well as towards the assessment of its spatial patterns, and the modelling of more brain atrophy indicators. 
Acknowledgements. This work was supported by the Inria Sophia Antipolis - Méditerranée, "NEF" computation cluster. This research has been conducted using the UK Biobank Resource undder Application Number 20576 (PI Nicholas Ayache). Additional information can be found at: https://www.ukbiobank.ac.uk

\section{References}

1. A. Ois et al. Heart failure in acute ischemic stroke. Journal of Neurology, $255(3): 385-389,2008$.

2. E. J. Benjamin et al. Heart disease and stroke statistics - 2018 update: A report from the american heart association. Circulation, 137(12), 2018.

3. M. R. Azarpazhooh et al. Concomitant vascular and neurodegenerative pathologies double the risk of dementia. Alzheimer's and Dementia, 14(2):148-156, 2018.

4. C. Sudlow et al. Ukbiobank an open access resource for identifying the causes of a wide range of complex diseases of middle and old age. PLoS Med., 12:1-10, 2015.

5. Simon R Cox et al. Associations between vascular risk factors and brain MRI indices in UK Biobank. bioRxiv, 2019.

6. L. O. Müller and E. F. Toro. Enhanced global mathematical model for studying cerebral venous blood flow. Journal of Biomechanics, 47(13):3361-3372, 2014.

7. S. Safaei et al. Bond graph model of cerebral circulation: Toward clinically feasible systemic blood flow simulations. Frontiers in Physiology, 9:1-15, 2018.

8. P. Blanco et al. An anatomically detailed arterial network model for onedimensional computational hemodynamics. IEEE Transactions on Biomedical Engineering, 62(2):736-753, 2015.

9. R. Molléro, X. Pennec, H. Delingette, N. Ayache, and M. Sermesant. Populationbased priors in cardiac model personalisation for consistent parameter estimation in heterogeneous databases. International Journal for Numerical Methods in Biomedical Engineering., 2018.

10. Alvaro Alonso and Antonio P Arenas de Larriva. Atrial Fibrillation, Cognitive Decline and Dementia. European Cardiology Review, 11(1):49, 2016.

11. Paul Schmidt. Bayesian inference for structured additive regression models forlarge-scale problems with applications to medical imaging. PhD thesis, LudwigMaximilians-Universität München, 2017.

12. F. Alfaro-Almagro et al. Image processing and quality control for the first 10,000 brain imaging datasets from uk biobank. NeuroImage, 166:400-424, 2018.

13. D. Chapelle, P. Le Tallec, P. Moireau, and M. Sorine. Energy-preserving muscle tissue model: formulation and compatible discretizations. International Journal for Multiscale Computational Engineering, 10(2):189-211, 2012.

14. M. Caruel, R. Chabiniok, P. Moireau, Y. Lecarpentier, and D. Chapelle. Dimensional reductions of a cardiac model for effective validation and calibration. Biomechanics and Modeling in Mechanobiology, 13:897-914, 2014.

15. N. Hansen. The cma evolution strategy: A comparing review. Towards a New Evolutionary Computation, 102:75-102, 2016.

16. R. Modir, H. Gardener, and C. B. Wright. Stroke blood pressure and white matter hyperintensity volume - a review of the relationship and implications for stroke prediction and prevention. US Neurology, 8(1):33-36, 2012.

17. Y. Seko et al. Association between atrial fibrillation, atrial enlargement, and left ventricular geometric remodeling. Scientific Reports, 8(1):1-8, 2018. 